\title{
FP-LAPW Study of the EFG at Impurity Sites in Oxides: Cd in Rutile $\mathrm{TiO}_{2}$
}

\author{
Leonardo A. Errico ${ }^{1}$, Gabriel Fabricius ${ }^{2}$, and Mario Rentería ${ }^{2}$ \\ Departamento de Física, Facultad de Ciencias Exactas, Universidad Nacional de La Plata, \\ C. C. $\mathrm{N}^{\circ} 67,1900 \mathrm{La}$ Plata, Argentina \\ ${ }_{2}^{1}$ Fellow of CONICET, Argentina \\ 2 Member of CONICET, Argentina \\ Reprint requests to Dr. G. F.; Fax: 54-221-4252006; E-mail: fabriciu@venus.fisica.unlp.edu.ar \\ Z. Naturforsch. 55a, 267-270 (2000); received August 26, 1999
}

Presented at the XVth International Symposium on Nuclear Quadrupole Interactions, Leipzig, Germany, July 25-30, 1999.

\begin{abstract}
We report here first-principles determination of the electric-field gradient (EFG) tensor at the Cd impurity located at cation sites in rutile $\mathrm{TiO}_{2}$. As far as we know, these represent the first $a b$ initio calculations at impurity sites with the FP-LAPW method in oxide systems. We used super-cells to simulate the diluted impurity in the crystal. The free-relaxation process performed in our study shows that the changes in distances of the oxygen nearest-neighbours to the impurity are not isotropic as was supposed in a previous study within the muffin-tin approximation. Our prediction for the EFG component of major absolute value agrees well with the experiment and is opposite in sign and direction if isotropic relaxations are assumed. Our value of the asymmetry parameter $\eta$ also compares very well with the experimental value.
\end{abstract}

Key words: Electric-Field Gradient; FP-LAPW; Impurity-Induced Lattice Relaxation; Electronic Structure; $\mathrm{TiO}_{2}$

\section{Introduction}

The electric-field gradient (EFG) tensor at crystalline sites in solids can be determined by several experimental techniques such as Perturbed-Angular Correlations (PAC), Nuclear Quadrupole Resonance (NQR), Perturbed Angular Distributions (PAD) and Mössbauer Spectroscopy (MS). Through the measurement of the EFG in adequate ionic sites in solids it is possible to obtain a nanoscopic and highly localised characterisation of disperse species or coexistent compound phases as well as to monitor phase transitions or direct populations of non-equivalent crystalline sites [1-4]. The study of the EFG dependence on temperature, pressure, impurity character, concentration, etc., enables the development of semiempirical and theoretical models that goes deeper in the understanding of the physical properties of condensed matter.

From the theoretical point of view, the determination of the EFG at an impurity has several difficulties that make a realistic calculation very cumbersome. The EFG tensor elements, $\mathbf{V}_{i j}$, are very sensitive to the asymmetry of the charge distribution in the neighbourhood of the impurity. This fact necessitates a very accurate electronicstructure calculation and also, as we will show, a very ac- curate estimation of the ionic relaxations introduced by the impurity in the host. As far as we know there have not been many attempts to treat these problem from firstprinciples. One of them is the work of Sato et al. [5], where a muffin-tin approximation is made for the potential and an isotropic relaxation is proposed for the oxygen nearest-neighbours $(\mathrm{NN})$ of the impurity $(\mathrm{Cd}$ in $\mathrm{TiO}_{2}$ ).

In this work we report a first-principles determination of the EFG tensor at a Cd impurity located at a Ti cation site in rutile $\mathrm{TiO}_{2}$. We used super-cells to simulate the impurity system and performed calculations of the electronic structure and forces on the atoms. We carried out a free-relaxation of the NN oxygen atoms of the impurity and obtained that in the system studied this approach gives an EFG having different sign and pointing in a different direction than that obtained if isotropic relaxations are assumed as was done by Sato et al. [5].

\section{Method of Calculation}

Our aim was to calculate the EFG tensor at a $\mathrm{Cd}$ impurity replacing $\mathrm{Ti}$ in rutile $\mathrm{TiO}_{2}$. The general scheme we adopted to deal with this problem was the following: we 
considered a periodically repeated super-cell containing a single $\mathrm{Cd}$ impurity and performed first-principles calculations in order to determine the self-consistent potential and charge density inside the cell. We studied the relaxation introduced by the impurity, computing the forces on the Cd oxygen nearest-neighbours (Cd-O NN) and moving them until the forces vanished. We followed during this study the behaviour of the EFG tensor, taking into account the charge state of the impurity, as we explain further below.

The calculations were performed with the WIEN97 implementation, developed by Blaha et al. [6], of the FullPotential Linearized-Augmented-Plane-Wave (FP-LAPW) method [7], and we worked in the LDA approximation [8]. In this method no shape approximation on either the potential or the electronic charge density is made, being thus specially suited for EFG calculations. For methodological purposes the unit cell is divided into non-overlapping spheres with radius $R_{i}$ and an interstitial region. The atomic spheres radii used for $\mathrm{Cd}, \mathrm{Ti}$ and $\mathrm{O}$ were 1.11 , 0.95 and $0.85 \AA$, respectively. We took for the parameter $R K_{\mathrm{MAX}}$, which controls the size of the basis-set in these calculations, the value 7 . This value gives well converged basis-sets consisting of 1,200 LAPW functions. We also introduced local orbitals (LO) to include Ti-3s and $3 p, O-2 p$ and Cd- $4 p$ orbitals. The correctness of the choice of these parameters was checked by performing calculations for other $R_{i}$ 's and $R K_{\mathrm{MAX}}$ 's. Integration in reciprocal space was performed using the tetrahedron method taking $150 \mathrm{k}$-points in the first Brillouin zone. Once self-consistency of the potential was achieved, quantum-mechanically-derived forces were obtained according to Yu et al. [9], and the ions were displaced according to an Newton damped scheme [10] until the forces on Cd-O NN's were below some tolerance value taken as $0.025 \mathrm{eV} / \AA$.

In the LAPW method, the $\mathbf{V}_{i j}$ are obtained directly from the $V_{2 \mathrm{M}}$ components of the lattice harmonic expansion of the self-consistent potential (a more detailed description of the formalism used to compute the EFG tensor may be found in [11]). The valence contribution to $\mathbf{V}_{i j}$ originates from the asymmetry of the valence electronic charge density and is the dominant contribution. Given that this term is so sensible to the details of the electronic structure, we reviewed at this point the approach used for the case of an impurity system, in our case the double-acceptor $\mathrm{Cd}^{2+}$ in $\mathrm{Ti}^{4+} \mathrm{O}_{2}^{2-}$. The consideration of a super-cell to simulate the impurity $\mathrm{Cd}$ in $\mathrm{TiO}_{2}$ implies that we are working in a metallic system, and the size of the super-cells considered in this work is small enough to

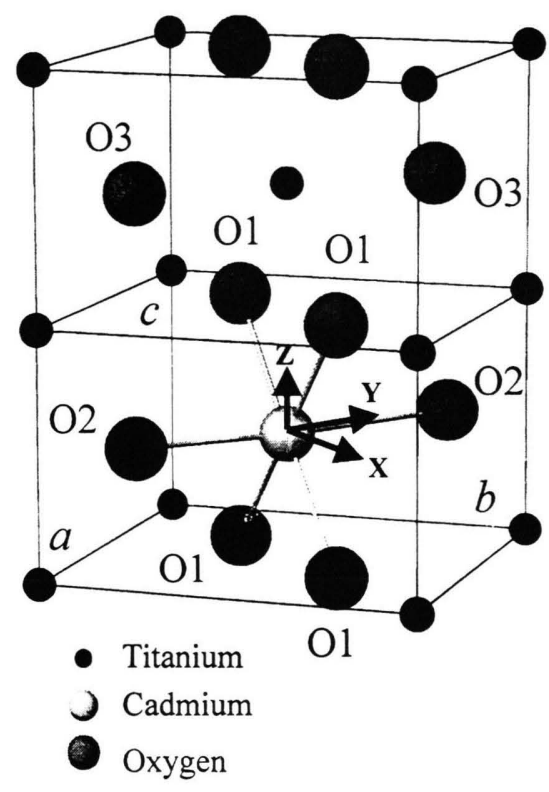

Fig. 1. Double super-cell used in the present work (see text). $\mathbf{V}_{i i}$ values throughout the article are referred to the principal axis system indicated in this figure.

make the valence contribution to $\mathbf{V}_{i j}$ dependent on the filling of the band. Then, at each step of the relaxation, once we have obtained the self-consistent potential, we computed again the valence charge density adding two electrons to the super-cell in order to fill up the p-oxygen band and mimic the physical situation of a highly diluted impurity system.

\section{Results and Discussion}

\subsection{Cell and Super-Cells}

The rutile $\left(\mathrm{TiO}_{2}\right)$ structure is tetragonal $(a=b=4.594 \AA$, $c=2.959 \AA$ ), the unit cell containing 2 metal atoms ( $\mathrm{Ti}$ ) at positions $2 \mathrm{a}(0,0,0)$ and $(1 / 2,1 / 2,1 / 2)$ and 6 anions (O) at positions $4 \mathrm{f}(1 \pm u, 1 \pm u, 0)$ and $(1 / 2 \pm u,-( \pm u-1 / 2)$, $1 / 2$ ) with $u=0.305$ [12]. The super-cell (called "doublecell") considered in the present work is shown in Figure 1. It consists of two units cells of $\mathrm{TiO}_{2}$ stacked along the $c$-axis with one body-centered $\mathrm{Ti}$ atom replaced by the $\mathrm{Cd}$ atom. Even when the concentration of $\mathrm{Cd}$ is absolutely unrealistic compared with ppm dilutions of PAC experiments, this super-cell has several adequate features for our purposes: the $\mathrm{Cd}$ atom has the same first two shells of nearest neighbours ( 6 oxygen and $8 \mathrm{Ti})$ as in the ideal situation of a high dilute impurity system; the same holds 


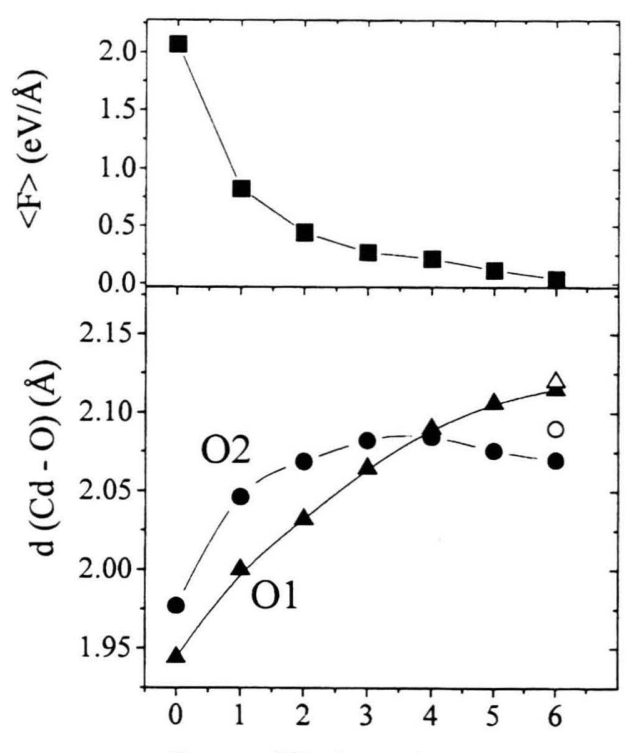

Step of Relaxation Process

Fig. 2. Quadratic mean force on $\mathrm{O} 1$ and $\mathrm{O} 2$ (top) and distances between $\mathrm{Cd}$ and oxygen atoms during the relaxation process (bottom). Open symbols are results of the relaxation in the "quadruple cell".

for the oxygen atoms to be relaxed that have one $\mathrm{Cd}$ and two Ti neighbours.

\subsection{Relaxation Process}

\section{(a) 12-atoms "double-cell"}

In order to study the relaxation introduced by the impurity we considered oxygen $\mathrm{O} 1, \mathrm{O} 2$, and $\mathrm{O} 3$ displacements until forces vanished (see Figure 1). Displacement of $\mathrm{O} 3$ atoms, which is very small, is not essential, and none of the conclusions of this work are affected by it, thus we will not mention it in what follows. In Fig. 2 we show the distance from $\mathrm{Cd}$ to $\mathrm{O} 1$ and $\mathrm{O} 2$ along the process of relaxation. As expected, the oxygen atoms relax outwards along the $\mathrm{Cd}-\mathrm{O}$ directions but our results show that the relaxation is far from being isotropic as Sato et al. have supposed working with the same super-cell [5]. In fact, $\mathrm{O} 2$ atoms are nearer to the impurity at the end of the process than $\mathrm{O} 1$ atoms. This means that the situation was inverted with respect to the initial configuration described in Section 3.1.

\section{(b) 24-atoms "quadruple-cell"}

In order to check that the relaxation obtained is not spurious effect of the "double-cell" (note that two $\mathrm{O} 2$ at-

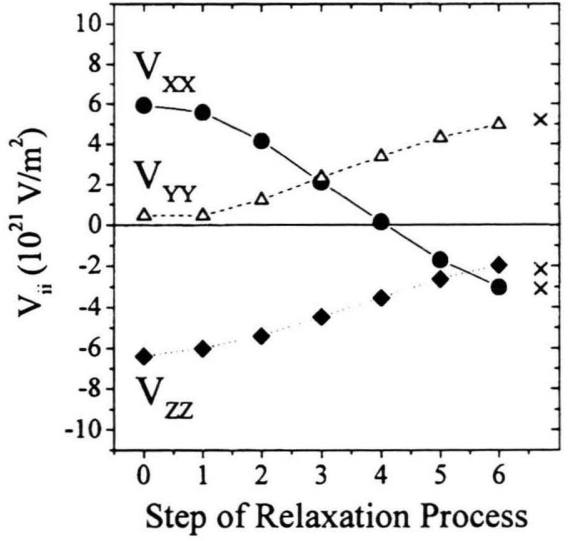

Fig. 3. Calculated EFGs at $\mathrm{Cd}$ in $\mathrm{TiO}_{2}$ in the "double-cell" of Fig. 1 during the relaxation process. Experimental values [13] are also shown (crosses), assuming our calculated sign for the major absolute value component.

oms from neighbouring cells approach each other since they are both neighbours of $\mathrm{Cd}$ atoms located along the (-110) direction) we have considered at 24-atoms supercell repeating the "double-cell" of Fig. 1 in the (010) direction but replacing the $\mathrm{Cd}$ atom by $\mathrm{Ti}$ and with all the atoms of the $\mathrm{TiO}_{2}$ structure in their original positions. In this way, the atoms that were in the $(-110)$ direction in the "double-cell" of Fig. 1 as: Cd-O2-O2-Cd are now as: $\mathrm{Cd}-\mathrm{O} 2-\mathrm{O} 2$ '- $\mathrm{Ti}$, where the $\mathrm{O}_{2}^{\prime}$ is also allowed to relax. In the first run we located $\mathrm{O} 1, \mathrm{O} 2$ and $\mathrm{O} 3$ at the relaxed positions of the 12-atoms "double-cell" and obtained that the forces were small, confirming that the results of the relaxation with the "double-cell" of Fig. 1 are, at least qualitatively, right. Two steps of further relaxation produced the changes in distances shown in Fig. 2 with open symbols.

\subsection{Electric-Field Gradients}

In every step of the relaxation process, $\mathbf{V}_{i j}$ is diagonal in the $x, y, z$ axis-system of Figure 1. In Fig. 3 we show the behaviour of the diagonalised tensor in this system, $\mathbf{V}_{i i}$, for all the steps of the 12-atoms "double-cell" relaxation. We see that, even when the displacements of oxygen atoms are small (Fig. 2), their effect on $\mathbf{V}_{i i}$ is quite strong. In particular, the major component of the EFG (the magnitude to be compared with a PAC experiment) changes it's sign and axis when the distance $\mathrm{Cd}-\mathrm{O} 1$ changes from being smaller to bigger than the distance $\mathrm{Cd}-\mathrm{O} 2$ (steps 3 to 5 in Figure 2). The values obtained for the greatest absolute component of the EFG (eq) and $\eta$ reproduce successfully the experimental values (see Ta- 
Table 1. Experimental and theoretical electric-field gradients for $\mathrm{Cd}$ in $\mathrm{TiO}_{2}$ in units of $10^{21} \mathrm{~V} / \mathrm{m}^{2}$. The sign and directions of the experimental values are unknown. eq denotes the component of major absolute value.

\begin{tabular}{llllll}
\hline & $V_{X X}$ & $V_{Y Y}$ & $V_{Z Z}$ & eq & $\eta$ \\
\hline Experiment [13] & & & & $5.23(5)$ & $0.18(1)$ \\
$\quad$ [14] & & & & $5.76(2)$ & $0.18(1)$ \\
FP-LAPW & & & & & \\
$\begin{array}{l}\text { Free-relaxation } \\
\text { Muffin-tin [5] }\end{array}$ & -3.0 & +5.0 & -2.0 & +5.0 & 0.2 \\
$\begin{array}{l}\text { Isotropic relax. } \\
\text { FP-LAPW }\end{array}$ & +1.54 & +3.56 & -5.09 & -5.09 & 0.39 \\
Isotropic relax. & +3.6 & +1.1 & -4.7 & -4.7 & 0.5 \\
\hline
\end{tabular}

ble 1). We are confident about the sign and direction of eq obtained in our calculation, but the absolute value and the value of $\eta$ are quite sensible to a small refinement in the oxygen positions. We think that a more accurate relaxation with bigger super-cells (that are in progress) are needed to make an accurate prediction of EFGs. It is interesting to note that the results obtained by Sato et al. [5] also agree with the available experimental information.

Their values for $\mathbf{V}_{i i}$ (see Table 1) are similar to the ones we obtain when $d(\mathrm{Cd}-\mathrm{O} 1)<d(\mathrm{Cd}-\mathrm{O} 2)$ (e.g., step 2 in Fig. 3), suggesting that the difference between the two calculations comes from the different approach to per-

[1] E. N. Kaufmann and R. Vianden, Rev. Mod. Phys. 51, 161 (1979).

[2] A. Lerf and T. Butz, Angew. Chem. Int. Ed. Engl. 26, 110 (1987).

[3] M. Uhrmacher, R. N. Attili, K. P. Lieb, K. Winzer, and M. Mekata, Phys. Rev. Lett. 76, 4829 (1996).

[4] M. Rentería, F. G. Requejo, A. G. Bibiloni, A. F. Pasquevich, J. Shitu, and K. Freitag, Phys. Rev. B55, 14200 (1997).

[5] K. Sato, H. Akai, and T. Minamisono, Z. Naturforsch. 53a, 396 (1998).

[6] P. Blaha, K. Schwarz, P. Dufek, and J. Luitz, Wien97, Vienna University of Technology 1997. Improved and updated Unix version of the original copyrighted Wien-code, which was published by P. Blaha, K. Schwarz, P. I. Sorantin, and S. B. Trickey, in Comput. Phys. Commun. 59, 399 (1990). form the relaxation. But, in fact, Sato et al. also use another approach to calculate the electronic structure, i.e. the KKR method with the muffin-tin approximation. Hence, in order to separate the reasons of disagreement, we calculated $\mathbf{V}_{i j}$ with our method and the oxygen atoms located at their suggested positions (isotropic relaxation). In this case we obtained the same sign and axis for the major component of the EFG than they did (see Table 1), confirming that the different predictions made by the two approaches are due to the different relaxation proposed. There is, however, an interchange between $V_{X X}$ and $V_{Z Z}$ that could be attributed to the muffin-tin approximation.

We therefore conclude that a free-relaxation treatment of the distorsions introduced by the impurity in its first shell of neighbours is crucial in order to make EFG predictions. An experimental determination of the sign and axis of eq would be very important to confirm the relevant features of the relaxation predicted in the present work. PAC experiments in single crystals are envisaged in our group in order to determine the axis of eq.

This work was partially supported by CONICET, Fundación Antorchas, CICpBA, and ANPCyT (PICT98 03-03727), Argentina, and The Third World Academy of Sciences (TWAS), Italy (RGA 97-057). Prof. Dr. M. Weissmann is kindly acknowledged for fruitful discussions and encouragement on this work.

[7] S. H. Wei and H. Krakauer, Phys. Rev. Lett. 55, 1200 (1985).

[8] J. P. Perdew and Y. Wang, Phys. Rev. B45, 13244 (1992).

[9] R. Yu, D. Singh, and H. Krakauer, Phys. Rev. B43, 6411 (1991).

[10] B. Kohler, S. Wilker, M. Scheffler, R. Kouba, and C. Ambrosch-Draxl, Comput. Phys. Commun. 94, 31 (1996).

[11] K. Schwarz, C. Ambrosch-Draxl, and P. Blaha, Phys. Rev. B42, 2051 (1990).

[12] R. Restori, D. Schwarzenbach, and J. R. Schneider, Acta Crystallogr. B43, 251 (1987).

[13] Th. Wenzel, A. Bartos, K. P. Lieb, M. Uhrmacher, and D. Wiarda, Ann. Physik 1, 155 (1992).

[14] J. M. Adams and G. L. Catchen, Phys. Rev. B50, 1264 (1994). 